\title{
Periodic table from the Baruton perspective: Some anomalies and spin correlation effect
}

\author{
Ali Bayri ${ }^{1}$, fatih bulut ${ }^{1}$, and Serdar Altin $^{1}$ \\ ${ }^{1}$ Inonu University
}

January 19, 2021

\begin{abstract}
In this study, we have looked the periodic table from the Barut's point of view and discussed the deviations from the Madelung rule. Expected, observed and computed total energies (Hartree-Fock and Gaussian) are given for two different (one for expected and the other one is observed) configurations of the $\mathrm{Cr}$ atom. The data shows that preferred electronic configuration for the $\mathrm{Cr}$ is $4 \mathrm{~s} 13 \mathrm{~d} 5$ not $4 \mathrm{~s} 23 \mathrm{~d} 4$ as dictated by the Madelung rule. This event may be due to the spin correlation effect which is closely related to the Hund's rule.
\end{abstract}

Periodic table from the Baruton perspective: Some anomalies and spin correlation effect

A. Bayri, F.Bulut and S. Altin

Inonu University, Physics Department, Malatya, Turkey

Abstract: In this study, we have looked the periodic table from the Barut's point of view and discussed the deviations from the Madelung rule. Expected, observed and computed total energies (Hartree-Fock and Gaussian) are given for two different (one for expected and the other one is observed) configurations of the Cr atom. The data shows that preferred electronic configuration for the $\mathrm{Cr}$ is $4 \mathrm{~s}^{1} 3 \mathrm{~d}^{5}$ not $4 \mathrm{~s}^{2} 3 \mathrm{~d}^{4}$ as dictated by the Madelung rule. This event may be due to the spin correlation effect which is closely related to the Hund's rule.

Keywords: Baruton, Madelung rule, spin correlation.

\section{Introduction}

Nearly 150 years ago an atomistic explanation of the periodic table had been given by Mandeleev [1]. Very recently a quantum aspect of the periodic table has been questioned [2]. As is already pointed out in that article, 5 scientists separately tried to explain the Madelung's rule with group theoretical approach $[3,4,5$, $6,7,8]$. Among these studies Fet's and Barut's approach were better in many aspect. In other words the dynamical Group theoretical explanation of the periodic system was mainly from $[4,5]$. We will not going to discuss the group theoretical studies in this study but in future work we would explain some observer anomalies from the periodic table by dynamical Group theoretical method. In this study we mainly focus on some numeric calculation together with some other work with the literature. Using the calculation we would try to explain some discrepancies from the expectations. Although we will study the group theoretical aspect of the problem in some future work, we should remember that Barut like Fet were forced to treat the element, not as composite particle, but as state of superparticle [2], where group structure was investigated on the basis of the group $\mathrm{SO}(4,2)$ which is also discussed by Odabasi [9]. These superparticles (supermultiplets) later named as Baruton [10]. By starting from the fact that a special representation of the dynamical group $\mathrm{SO}(4,2)$ provides the quantum numbers $\mathrm{n}, \mathrm{l}, \mathrm{m}$ for the hydrogen atom [11] and by taking each atom or ion 
in its normal configuration as a state, Barut demonstrated that hydrogenic order can be explained by the chain

$\mathrm{SO}(4,2)-\mathrm{SO}(4,1)-\mathrm{SO}(4)-\mathrm{SO}(3)$

While Madelung rule is given by the reductions,

$\mathrm{SO}(4,2)-\mathrm{SO}(3,2)-\mathrm{SO}(3) \mathrm{XSO}(2)$

Barut also showed that $[4,5]$ increasing order of element does not always corresponds to the increasing order of energy in the hydrogen atom. In the Hydrogen atom the energy is increased with increasing $\mathrm{n}$ and when relativistic spin-orbit is included it increases with 1 , and this rule is generally denoted $(\mathrm{n}+\mathrm{l}, \mathrm{n})$ rule $[4$, 5]. From the Barut's work it is important to figure out that for ions, the electron shells are also filled in different order from that for atoms, the hydrogenic order being the limit for highly ionized case. In future work we would try to apply the Barut's method for some specific unexpected filling. In this study we mainly concentrate on some unusual filling especially filling up the $\mathrm{Cr}$ atom by calculating the self-consistent field. As is pointed out earlier, the filling up principle was discovered empirically by Madelung [12] for atoms as:

1-When consecutive atoms are considered, the electron shells are filled in the order of the sum of two quantum numbers $\mathrm{n}$ and $\mathrm{l}$, that is, $(\mathrm{n}+\mathrm{l})$.

2-Shelss with equal $\mathrm{n}+\mathrm{l}$ numbers are filled in the order of quantum number $\mathrm{n}$.

This rule is very successful for how the energy levels are filled for the neutral atoms. However experiments show that there are some deviations from this rule.

Madelung's second rule predicts that the $4 \mathrm{~s}$ shell will be filled before $3 \mathrm{~d}$ shell and that $4 \mathrm{f}$ shell and $5 \mathrm{f}$ shell would be occupied first at $\mathrm{Z}=57$ and $\mathrm{Z}=89$ respectively. From the atomic spectroscopic data it is quite evident that there are same expectations for this rule. For example for $\mathrm{Cr}$ atom the expected occupation is $4 \mathrm{~s}^{2} 3 \mathrm{~d}^{4}$ but the correct occupation was $4 \mathrm{~s}^{1} 3 \mathrm{~d}^{5}$. In this study we mainly concentrate on this anomaly and try to find out why the second configuration lies lowest. The energies of the $3 \mathrm{~d}$ and $4 \mathrm{~s}$ states are so close together that small effects can shift the balance either way $[4,5]$. It is assumed that by putting four electrons into the $3 \mathrm{~d}$ state their repulsion raises $4 \mathrm{~s}$ level such that one electron shifts over. The requirement of parallel spins for electrons that do occupy different orbitals is a consequence of a quantum mechanical effect called spin correlation effect with the tendency for two electrons with parallel spins to stay apart from one another and hence to repel each other less. One consequence of this effect is that half-filled shells of electrons with parallel spins are particularly stable. For example, the ground state of the chromium atom is $4 \mathrm{~s}^{1} 3 \mathrm{~d}^{5}$ rather than $4 \mathrm{~s}^{2} 3 \mathrm{~d}^{4}$. An additional feature, another consequence of spin correlation, is that in some cases a lower total energy may be obtained by forming a half-filled or filled d subshell, even though that may mean moving an s electron into the d subshell. Therefore, as a half-filled d shell is approached the ground-state configuration is likely to be $\mathrm{d}^{5} \mathrm{~s}^{1}$ and not $\mathrm{d}^{4} \mathrm{~s}^{2}$ (as for $\mathrm{Cr}$ ).

\section{Theoretical Calculation of Ground term for the Cr Atom}

As we pointed out earlier there are some discrepancies from the expected filling of the Madelung prediction for half-filled and full filled $\mathrm{d}$ and $\mathrm{f}$ shells. Here we mainly concentrate on the $\mathrm{Cr}$ atom where the expected filling is $4 \mathrm{~s}^{2} 3 \mathrm{~d}^{4}$ but the true occupation is $4 \mathrm{~s}^{1} 3 \mathrm{~d}^{5}$. Here we mainly tried to find out the energy differences of these two different configurations by self consistently. It is obvious that the term symbol for $4 \mathrm{~s}^{2} 3 \mathrm{~d}^{4}$ and $4 \mathrm{~s}^{1} 3 \mathrm{~d}^{5}$ would be ${ }^{5} \mathrm{D}$ and ${ }^{7} \mathrm{~S}$ respectively.

Many authors have calculated the Hartree-Fock energy of these two different configuration in order to work out the deviation from the Madelung rule. Hartree-Fock is the usual method for exploiting the exceptions of the Madelung rule. Apart from the literature, we have used the Gaussian Software to compute the total energies of the electronic configurations of the $\mathrm{Cr}$ atom. In table 1 we have compared those works in the literature with our results.

\section{Theoretical Central Field Calculation of Madelung Rule}


Many of the most important quantum mechanical systems involve atoms or molecules which must be solved numerically since there is no analytical solution with many body interactions. As can be pointed out with many authors $[9,13]$ these problems involve a number of electrons around a number of atomic nuclei (only for one nuclei for atoms). Unfortunately, a full quantum solution of such a system of any nontrivial size is very difficult [14]. As discussed with T. Kago et al in order to find any correlation with the spectroscopic results, some approximation can be made. One of such approximation is the Hartree Fock approximation. We are not going to discuss the way of calculation but a good review of how to apply Hartree Fock approximation is given by [15]. By using gaussian type molecular orbital we have calculated the energies of two different configurations. It is quite clear that our results are in good agreement with the literature which is displayed in table 1.

Table1: $E$ is the deviation from the Madelung rule (energies are in a.u.)

\begin{tabular}{llll}
\hline Representation & calculated energy & References & $E$ (Energy gain) \\
\hline${ }^{5} \mathrm{D}$ & -1043.25809553 & Our work & -0.0408024 \\
${ }^{7} \mathrm{~S}$ & -1043.29889793 & Our work & \\
${ }^{5} \mathrm{D}$ & -1043.3082 & Odabasi [9] & -0.0471 \\
${ }^{7} \mathrm{~S}$ & -1043.3553 & Odabasi [9] & -0.0443 \\
${ }^{5} \mathrm{D}$ & -1043.0891 & Fischer [13] & \\
${ }^{7} \mathrm{~S}$ & -1043.1334 & Fischer [13] & (numerical Hartee fock) [14] \\
${ }^{7} \mathrm{~S}$ & -1043.356376 & &
\end{tabular}

\section{Discussion}

In this study we mainly concentrated on the calculation of the Self consistent field calculation for $\mathrm{Cr}$ atom where the expected configuration is different from the observed one. As is already explained in the text the observed ground term and the expected ones are ${ }^{7} \mathrm{~S}$ and ${ }^{5} \mathrm{D}$ respectively. The total energy values for these representations determine the relative position of the states of one configuration to those of another. It is obvious from the table 1 that all calculations suggest the ${ }^{7} \mathrm{~S}$ representation should lie lowest which represents a deviation from Madelung's rule.

The interaction between the nd orbitals and the core-electron orbitals might be the reason for this exception, the shells are seems to be filled according to the hydrogenic rule rather than the first part of Madelung's rule, when there are more than four ad-shell electrons the quantum number $\mathrm{n}$ might have the dominant role.

Cr atom's electron configuration that is different than the Madelung's rule might be not only due to the Hund rules, but also with rhe phenomena known as 'spin correlation'. This effect should not be considered with itself, it should be taken into account with the Hund's first rule which states that the lowest energy atomic state is the one that maximizes the total spin quantum number for the electrons in the open subshell.

\section{Conclusion}

In this study, we have mainly tried to look the periodic table from the Barut's point of view and discussed the deviations from the Madelung rule $[4,5]$. This deviation is expected due to the reason called spin correlation effect which is mainly related to the Hund's first rule. As explained in the calculation section the expected, observed and computed total energies (Hartree-Fock and Gaussian) are given for two different (one for expected and the other one is observed) configurations of the $\mathrm{Cr}$ atom. Our results and the results calculated by other researcher showed that the preferred electronic configuration for the $\mathrm{Cr}$ is $4 \mathrm{~s}^{1} 3 \mathrm{~d}^{5}$ not $4 \mathrm{~s}^{2} 3 \mathrm{~d}^{4}$ as dictated by the Madelung rule. This event may be due to the spin correlation effect which is closely related to the Hund's rule.

\section{References}

1. Mandeleev, D.I., J. Russ. Phys, Chem. Soc. 1, 60, (1869). 
2. Thyssen, P., Ceulemans, A. (2020) Particular Symmetries: Group Theory of the Periodic System. Substantia 4(1): 7-22. doi: 10.13128/Substantia-671

3. Fet, A. I. (2016). Group Theory of Chemical Elements. Berlin: De Gruyter.

4. Barut, A. O. (1972a). Group Structure of the Periodic System. In: The Structure of Matter: Rutherford Centennial Symposium. Edited by B. G. Wybourne. Christchurch, New Zealand: University of Canterbury Press.

5. Barut, A. O. (1972b). Dynamical Groups and Generalized Symmetries in Quantum Theory. Edited by A. N. Brooks. University of Canterbury, Christchurch, New Zealand: Bascands Ltd

6. Novaro, O. (2006). Group Theory of the Periodic Table. In: The Mathematics of the Periodic Table. Edited by D. H. Rouvray and R. B. King. New York: Nova Science Publishers.

7. Ostrovsky, V. N. (2004). The Periodic Table and Quantum Physics. In: The Periodic Table: Into the 21st Century. Edited by D. H. Rouvray and R. B. King. Baldock, UK: Research Studies Press.

8. Byakov, V. M.; Kulakov, V. I.; Rumer, Y. B.; Fet A. I. (1976). Group-Theoretical Classification of Chemical Elements: I. Physical Foundations. Moscow: Preprint of the Institute of Theoretical and Experimental Physics, ITEP, 26.

9. Odabasi H. Int. J. Quan. Chem. Symp. 7,23, (1973)

10. C.C. Walhman, dynamical groups in Atomic and molecular physics, In: Recent Advances in group theory and their application in spectroscopy. Ed. J.C. Donini, New york plenum press. 1978 pp329403

11. A.O. Barut and H. Kleineit, Phys. Rev. 156, 1541(1967), 157,1180(1967)

12. E. Madelung, Mathematische, Hilfsmittel, des physicers, 3rd. Ed. (Springer, Berlin, 1936) 9359

13. Fischer C. F., Some Hartree-Fock Results for $(3 d+4 s) q+1$ Configurations, Canadian Journal of Physics. 49, 1205 (1971)

14. T. Koga et al. Chemically reliable uncontracted Gaussian-type basis sets for atoms $\mathrm{H}$ to Lr, Chemical Physics Letters 328 (2000) 473-482

15. Pablo Echenique and J. L. Alonso, Molecular Physics 105 (23-24), 3057-3098 (2007) 